\title{
Multi-Epitope Fusion Protein Eg mefAg-1 as a Serodiagnostic Candidate for Cystic Echinococcosis in Sheep
}

\author{
Liu Tianli ${ }^{1, \dagger}$, Wang Xifeng ${ }^{1, \dagger}$, Tian Zhenzhong ${ }^{1, \dagger}$, Wang Lixia', Zhang Xingxing ${ }^{2}$, Qiao Jun', Meng Qingling,", \\ Gong Shasha', Chen Ying', Cai Xuepeng ${ }^{3}$ \\ ${ }^{1}$ College of Animal Science \& Technology, Shihezi University, Shihezi, Xinjiang 832003, China; ${ }^{2}$ State Key Laboratory for Sheep Genetic Improvement \\ and Healthy Production, Xinjiang Academy of Agricultural and Reclamation Science, Shihezi, Xinjiang 832000, China; ${ }^{3}$ State Key Laboratory of \\ Veterinary Etiological Biology, Lanzhou Veterinary Research Institute, Chinese Academy of Agricultural Sciences, Lanzhou,
}

Gansu 730046, China

\begin{abstract}
Cystic echinococcosis (CE) in sheep is a hazardous zoonotic parasitic disease that is caused by Echinococcus granulosus (Eg). At present, serological test is an important diagnostic method for Eg infection in domestic animals. Here, a fusion protein Eg mefAg-1 harboring 8 dominant B-cell epitopes of Eg such as antigen B, tetraspanin 1, tetraspanin 6, reticulon and Eg95 was produced in $E$. coli and evaluated for CE in sheep by indirect ELISA. Eg mefAg-1 showed in ELISA a high sensitivity (93.41\%) and specificity (99.31\%), with a coincidence rate of $97.02 \%$. Overall, it is suggested that the Eg mefAg-1 could be a potential antigen candidate for CE serodiagnosis in sheep.
\end{abstract}

Key words: multi-epitope, fusion protein, serodiagnostic, antigen, cystic echinococcosis, indirect ELISA, sheep

Echinococcosis, also known as cystic echinococcosis (CE), is a zoonotic parasitic disease that is caused by the growth of larvae of Echinococcus granulosus (Eg) in the liver, lung and other organs of the host such as sheep and human beings [1-3]. This disease is distributed worldwide, across the 5 continents $[4,5]$. It has been found in 21 provinces or autonomous regions of China, especially serious in livestock of Xinjiang, Gansu, Ningxia, Qinghai, Tibet, Sichuan and Inner Mongolia [6-8]. It has been reported that the average infection rate of sheep CE in Xinjiang area is 30-50\% $[9,10]$. Animal CE seriously affected the production and development of livestock, causing great loss in livestock production and bringing enormous danger to the health of pastoralists [11,12].

Screening and preparation of highly specific and sensitive Eg antigen is the premise of the development of CE serological diagnosis [13-15]. Over the past years, diagnosis of CE mainly depends on serological detection of the intermediate host (especially in sheep) using cyst fluid, protoscolex protein or re-

- Received 1 May 2018, revised 13 January 2019, accepted 30 January 2019.

*Corresponding author (xjmqlqj@sina.com)

(c) 2019, Korean Society for Parasitology and Tropical Medicine

This is an Open Access article distributed under the terms of the Creative Commons Attribution Non-Commercial License (http://creativecommons.org/licenses/by-nc/4.0) which permits unrestricted non-commercial use, distribution, and reproduction in any medium, provided the original work is properly cited. combinant proteins as coating antigens [16-23]. Purified cyst fluid antigen is highly sensitive. However, it is affected by the developmental stage of the worm, resulting in differences in sensitivity and specificity in serological diagnosis [24]. Moreover, the purification process has not been standardized thus purified cyst fluid cannot be prepared in large scale, which limits the application of cyst fluid antigen $[16,20,23]$. By contrast, the use of recombinant protein for antibody detection displays good specificity $[13,25]$, however, sensitivity is lower than that of fluid antigen. Therefore, development and preparation of CE recombinant antigens with high reactionogenicity is the key to enhancing the diagnostic performance of $\mathrm{CE}$ serodiagnosis.

The aim of this study is to develop and evaluate a multi-epitope fusion protein Eg mefAg-1 for serodiagnosis of CE in sheep. Here, we constructed and expressed multi-epitope fusion cDNA Eg mefAg-1 in E. coli, purified and evaluated the specificity and reactionogenicity of the recombinant protein Eg multiepitope fusion Ag-1 (reEg mefAg-1) via indirect ELISA for CE serodiagnosis in sheep.

A total of 235 slaughtered sheep were collected from 6 different pastures of Shawan County in Xinjiang China, where is the endemic region of $\mathrm{CE}$ in sheep.

The blood samples were collected and marked with the animal's identification number before slaughter. Then sera of 
these sheep were respectively prepared, numbered and stored at $4^{\circ} \mathrm{C}$. The internal organs (especially lung and liver) of carcasses of sheep were examined for parasites. Those parasites were confirmed by PCR amplification of mitochondrial COX1, nad1, and nad5 genes and further verified by sequencing. The corresponding sera from infected sheep were labelled as positive or negative for $\mathrm{CE}$ according to the post-mortem examination result. Of 235 sera, 91 were positive for $\mathrm{CE}$, 5 were positive for Echinococcus multilocularis, 8 were positive for Cysticercus tenuicollis (Ct) and 8 were positive for Fasciola hepatica. These sera were separated by centrifugation at 2,000 $\times \mathrm{g}$ for $15 \mathrm{~min}$, aliquotted and preserved at $-20^{\circ} \mathrm{C}$ until used.

The linear antigenic epitopes of antigen $\mathrm{B}(\mathrm{AgB})$, tetraspanin 1 (TSP1), tetraspanin 6 (TSP6), reticulon (RTN4), and Eg95 proteins were predicted using ABCpred (http://www.imtech. res.in/raghava/abcpred/), BepiPred 1.0 Server (http://www.cbs. dtu.dk/services/BepiPred/), Immunomedicine Group (http:// imed.med.ucm.es/Tools/antigenic.pl) and DNAStar-Protean software [25]. Eight dominant antigenic epitopes were selected based on the prediction results (Table 1). The multi-epitope fusion CDNA Eg mefAg-1 was obtained by connecting the nucleotide sequences that were corresponding to the amino acid sequences of dominant epitopes by a flexible peptide coding sequence in certain order. The multi-epitope fusion cDNA Eg mefAg- 1 was analyzed by codon prediction software Racc (http://nihserver.mbi.ucla.edu/RACC/) and subsequently optimized to preferred codons of $E$. coli by synonymous mutations, and sent to Beijing Genomics Institute (BGI, Shenzhen, China) for cDNA synthesis.

Primer 5.0 software was used to design specific primers for synthesized multi-epitope fusion cDNA Eg mefAg-1. Forward primer P1: 5'-CCGGAATTCATGGTGGTAAAAAAACGT-3' (underline indicates $E c o \mathrm{R}$ I restriction site), reverse primer P2: 5'-CCGCTCGAGTTACATTACAGTGCTTTCC-3' (underline Xho I restriction site). Amplification conditions: pre-denaturation at $95^{\circ} \mathrm{C}$ for $5 \mathrm{~min}, 30$ cycles of denaturation at $94^{\circ} \mathrm{C}$ for $40 \mathrm{sec}$ annealing at $60^{\circ} \mathrm{C}$ for $40 \mathrm{sec}$, extension at $72^{\circ} \mathrm{C}$ for $50 \mathrm{sec}$ and final extension at $72^{\circ} \mathrm{C}$ for $10 \mathrm{~min}$. The PCR product was recovered and double digested by the restriction endonuclease EcoR I and Xho I (TaKaRa, Tokyo, Japan) together with pET-28a (+) plasmid (Invitrogen, Carlsbad, California, USA). The target fragment and the vector fragment were recovered individually and ligated using T4 DNA ligase (TaKaRa) at $4^{\circ} \mathrm{C}$ overnight, and then the ligation product was then transformed into competent E. coli BL21(DE3) cells (TaKaRa). The recombinant plasmid pET-meAg-1 extracted from $E$. coli was verified by PCR and double nuclease digestion.

The positive clone was inoculated into liquid LB medium (Difco, Detroit, Michigan, USA) and cultured at $37^{\circ} \mathrm{C}$ until $\mathrm{OD}_{600} \mathrm{~nm}$ reached 0.6-0.8. IPTG (TaKaRa) was then added at a final concentration of $1.0 \mathrm{mmol} / \mathrm{L}$ to induce the cDNA expression. The bacterium was harvested at $4 \mathrm{hr}, 6 \mathrm{hr}$, and $8 \mathrm{hr}$ following IPTG addition. The expressed recombinant Eg multiepitope fusion Ag-1 (Eg mefAg-1) was analyzed by SDS-PAGE. Meanwhile, Western blot analysis was preformed using sheep Eg positive serum as the primary antibody, and horseradish peroxidase (HRP) labeled rabbit anti-sheep IgG (EarthOx, San Francisco, California, USA) was used as the secondary antibody.

Briefly, $50 \mathrm{ml}$ of culture medium was centrifuged to collect the bacteria. The bacteria were resuspended in $25 \mathrm{ml}$ of binding buffer and ultra-sonicated until the solution was clear. The solution was centrifuged, and the supernatant was collected and filtered through a $0.22 \mu \mathrm{m}$ filter. The processed product was purified by passing through Ni-NTA affinity column (Invitrogen). After protein concentration determination, the Eg mefAg- 1 was aliquoted and stored at $-80^{\circ} \mathrm{C}$ until used.

Indirect ELISA was established according to Baoumi et al.'s method [20] with appropriate adjustments. Briefly, the Eg mefAg-1 was diluted to $4.0 \mu \mathrm{g} / \mathrm{ml}$ with $0.05 \mathrm{~mol} / \mathrm{L} \mathrm{pH} 9.6$ car-

Table 1. Epitope sequences selected from different antigen proteins of Echinococcus granulosus

\begin{tabular}{lrr}
\hline Protein & \multicolumn{1}{c}{ Epitope sequences } & Position \\
\hline AgB1 (HM357141) & DDGLTSTSRSVMKMFGEVKYFFERDPLGQKWVLLEEL & $1-38$ \\
AgB2 (HM357142) & WKKRWGELRDFFRND & $11-26$ \\
AgB4 (HM357144) & RDFFRSDPLGQK & $19-30$ \\
AgB4 (HM357144) & LEEEDEDDLK & $61-70$ \\
Eg95 (AF465599) & IETPRAGKKESTVM & $120-133$ \\
TSP1 (KM459009) & TGNCDQGKAQSANVTG & $195-210$ \\
TSP6 (XP_024355367) & TQAITAPDLTTPYPSS & $150-165$ \\
RTN4 (KM459011) & KFTEKPKREWR & $185-195$ \\
\hline
\end{tabular}


bonate buffer, aliquoted to the 96-well microtiter plate (Costar, Cambridge, Massachusetts, USA) at $100 \mu \mathrm{l} /$ well, and coated at $4^{\circ} \mathrm{C}$ overnight. On the next day, the coated plate was washed with $0.05 \mathrm{~mol} / \mathrm{L}$ PBST (pH 7.4, containing $0.5 \mathrm{ml} / \mathrm{L}$ Tween-20) 3 times, blocked with $10 \mathrm{~g} / \mathrm{L} \mathrm{BSA}(100 \mu \mathrm{l} /$ well $)$ at $37^{\circ} \mathrm{C}$ for $2 \mathrm{hr}$ and washed 3 times with PBST. The serum sample was 1:100 diluted with dilution buffer, added to each well $(100 \mu \mathrm{l} /$ well $)$ and incubated at $37^{\circ} \mathrm{C}$ for $1 \mathrm{hr}$, followed by washing 3 times with PBST. Then, plates were treated with horseradish peroxidase-conjugated rabbit-anti-sheep IgG (Abcam, Cambridge, England, UK) in optimal dilution 1:10,000 for $1 \mathrm{hr}$. After repeated washing, $50 \mu \mathrm{l} /$ well chromogenic substrate solution was added to the plate and incubated at $37^{\circ} \mathrm{C}$ in dark for 15 min. The reaction was terminated by $50 \mu \mathrm{l} /$ well $2 \mathrm{M} \mathrm{H}_{2} \mathrm{SO}_{4}$. The $\mathrm{OD}_{450} \mathrm{~nm}$ value of each well was determined by an enzymelinked detector (Biotek, Winooski, Vermont, USA), with the blank well as the negative control.

A total of 30 negative serum samples from sheep were subjected to Eg mefAg-1-ELISA. The $\mathrm{OD}_{450} \mathrm{~nm}$ value was determined and the data were analyzed using Excel 2000 and SPSS 17.0 software for Windows (Release 17.0 standard version, SPSS Inc.,
Chicago, Illinois, USA). The average (X) and standard deviation (SD) were calculated and $\mathrm{X}+3 \mathrm{SD}$ was considered as cut-off value to discriminate positive and negative samples.

The established indirect ELISA based on Eg mefAg-1 was used to evaluate the specificity by detecting 30 anti-Eg positive sera, 5 anti-Echinococcus multilocularis positive sera, 8 anti-Cysticercus tenuicollis $(\mathrm{Ct})$ positive sera, 8 anti-Fasciola hepatica positive sera and 30 anti-Eg negative sera to determine whether they have cross-reaction with Eg mefAg-1. A total of 235 sheep serum samples were subjected to the detection of Eg antibodies by Eg mefAg-1 and the commercial anti-Echinococcus IgG ELISA Kit (ab108733, Abcam, Cambridge, Massachusetts, USA). Thereinto, HRP labeled rabbit anti-sheep IgG (EarthOx) was used as the secondary antibody in the test. The sensitivity, specificity and agreement of the Eg mefAg-1-ELISA were calculated according to the true-positive and true-negative in $2 \times 2$ tables $[17,20]$. Sensitivity $(\%)=$ [true positive/(true positive+false negative) $] \times 100$; Specificity $(\%)=[$ true negative $/$ (true negative+false positive $)] \times 100$; Agreement $(\%)=[$ (true positive+true negative)/total number of samples] $\times 100$.

A 468 bp fragment of Eg mefAg-1 cDNA was successfully

$\mathrm{AgB} 2$

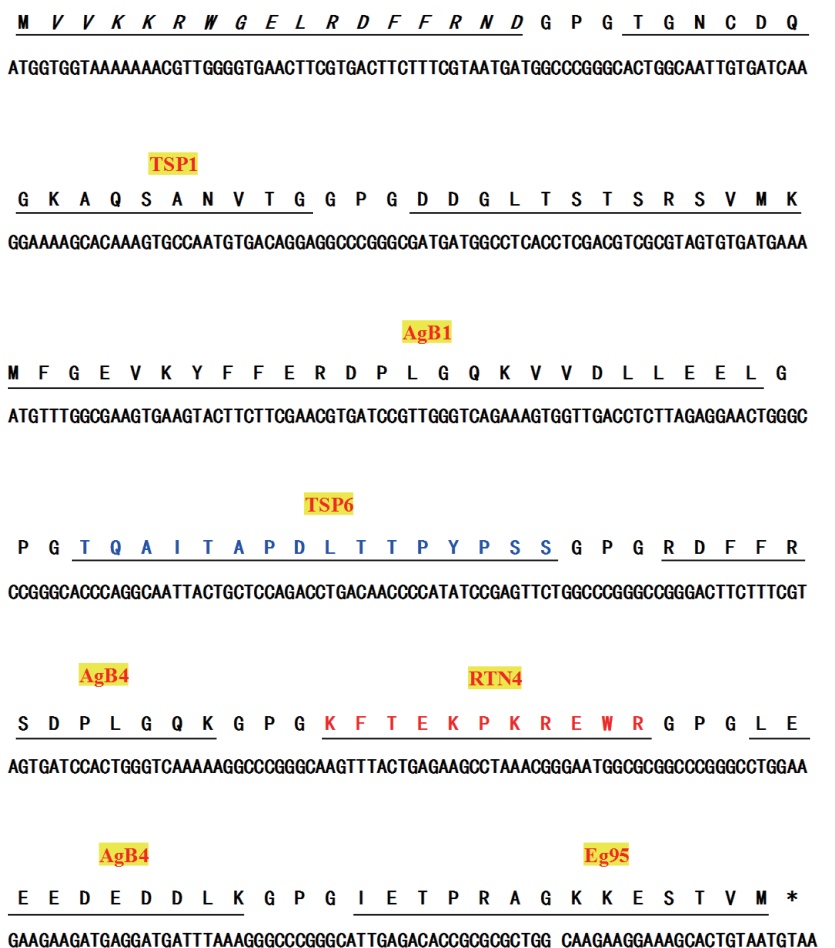

Fig. 1. Amino acid sequence of Eg mefAg-1 and its E. coli-codon optimized nucleotide sequence. A boxed GPG is linker. 
synthesized according to optimized nucleotide sequence of Eg mefAg-1 (Fig. 1). Then the cDNA was amplified using P1-P2 specific primers and cloned into pET28a (+) to generate recombinant plasmid pET-meAg-1. The pET-meAg-1 was subsequently verified by PCR amplification and double enzyme digestion, respectively, which produced target cDNA fragment with expected size, indicating the successful construction of the recombinant pET-meAg-1.

The results of SDS-PAGE revealed that positive clone expressed a $23.5 \mathrm{kDa}$ protein, consistent with expected size of Eg mefAg-1. The analysis of Western blot confirmed that recombinant protein Eg mefAg-1 purified by Ni-NAT affinity chromatography could be specifically recognized by anti-Eg positive serum (Fig. 2).

Indirect ELISA based on Eg mefAg-1 antigen indicated that the optimum coating amount of the antigen was $4.0 \mu \mathrm{g} / \mathrm{ml}$ and the best serum dilution was 1:100. The $\mathrm{OD}_{450 \mathrm{~nm}}$ values of the 30 Eg negative serum samples were mainly distributed at a range of 0.15-0.20. Statistical analysis showed that the average $(\mathrm{X}) \mathrm{OD}_{450}$ $\mathrm{nm}$ value of the negative serum samples was 0.159 and the standard deviation (SD) was 0.037. Accordingly, the confidence interval upper cut-off value was 0.270 . Samples with $\mathrm{OD}_{450} \mathrm{~nm}$ val-

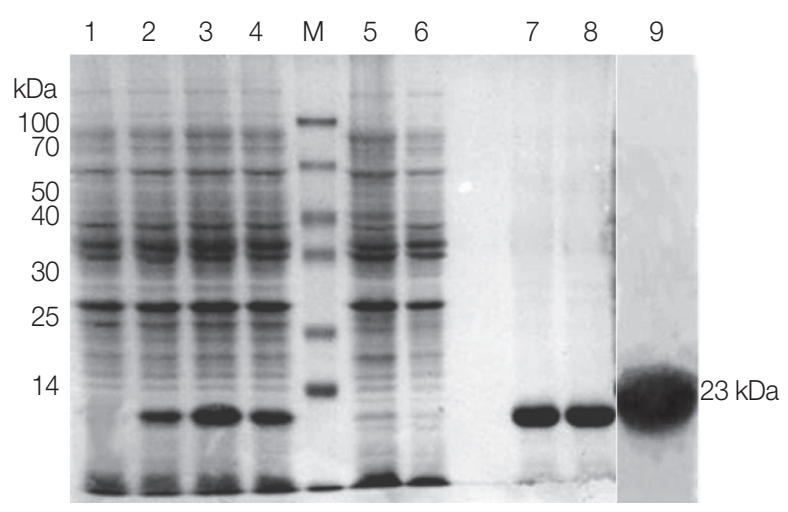

Fig. 2. Induction and purification of reEg mefAg-1 on SDS-PAGE gel and Western blotting. Mr, protein molecular weight marker; lane 1, culture supernatant of E. coli BL21(DE3); lanes 2-4, IPTGinduced for 4, 6, and $8 \mathrm{hr}$; lanes 5-6, pET-28a (+) vector (control); lanes 7-8, purified reEg mefAg-1 protein; lane 9, western blot with anti-Eg positive serum. ue greater than 0.270 were considered as positive, whereas less than or equal to 0.270 were considered as negative.

Specificity tests of Eg mefAg-1-ELISA showed that the 30 sheep Eg positive sera were positive and those positive sera against other parasites were negative, indicating that the Eg mefAg-1 was highly specific and had no cross-reactivity with positive sera against other parasites (Fig. 3).

A total of 235 sheep sera were detected for Eg antibody by Eg mefAg-1-ELISA. Of 235 sera, 86 were CE positive for Eg mefAg-1-ELISA method $(86 / 235,36.60 \%)$, whereas 89 were CE positive for commercial anti-Echinococcus IgG ELISA Kit $(89 / 235,37.87 \%)$. Out of 91 positive sera confirmed by necropsy, however, 6 sera were judged as negative by developed Eg mefAg-1-ELISA method (Table 2). Compared with the results of necropsy, Eg mefAg-1- ELISA presents high sensitivity (93.41\%, 85/91) and specificity $(99.31 \%, 143 / 144)$. Accordingly, the agreement of between Eg mefAg-1-ELISA and the necropsy was $97.02 \%$ (228/235) (Table 2). Moreover, when compared with the commercial anti-Echinococcus IgG ELISA Kit (ab108733, Abcam, USA), there were little discrepancy between the commercial anti -Echinococcus IgG ELISA Kit and the developed ELISA among individual sera.

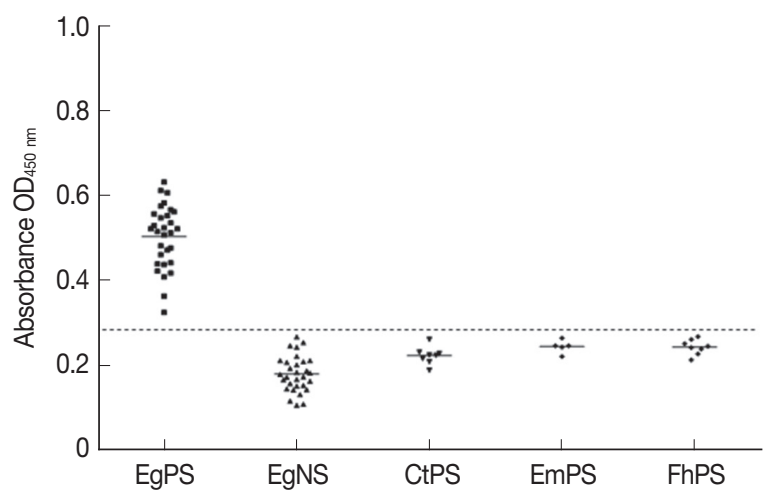

Fig. 3. Antigenicity of Eg mefAg-1 toward sheep hydatidosis sera in ELISA. A cut-off line was set at OD 0.270. Eg PS, E. granulosus hydatid cyst positive; Eg NS, E. granulosus hydatid cyst negative; Ct PS, Cysticercus tenuicollis positive; Em PS, E. multilocularis cyst positive; Fh PS, F. hepatica positive.

Table 2. Comparison among the results of necropsy, commercial anti-Echinococcus IgG ELISA Kit and Eg mefAg-1-ELISA in sheep sera

\begin{tabular}{|c|c|c|c|c|c|c|}
\hline & & \multicolumn{2}{|c|}{ Necropsy } & \multicolumn{2}{|c|}{$\begin{array}{c}\text { Commercial anti-Echinococcus } \\
\text { IgG ELISA Kit }\end{array}$} & \multirow{2}{*}{ Total } \\
\hline & & Positive & Negative & Positive & Negative & \\
\hline \multirow[t]{3}{*}{ Eg mefAg-1-ELISA } & Positive & 85 & 1 & 84 & 2 & 86 \\
\hline & Negative & 6 & 143 & 5 & 144 & 149 \\
\hline & Total & 91 & 144 & 89 & 146 & 235 \\
\hline
\end{tabular}


The cyst fluid (CF) and protoscolex proteins of Eg had been evaluated and proved to be valuable antigens for the CE serological diagnosis $[16,22,24]$. However, sensitivities and specificities of these antigen proteins differ greatly, and some of the cyst fluid antigen components such as metabolites and host tissue components can cross-react with nonspecific antibodies that target other worms, resulting in poor specificity [15,22]. Golassa et al. [17] evaluated the diagnostic value of crude cyst fluid in bovine CE via indirect hemagglutination test (IHA) and found that the false positive and false negative rates were high, and the accuracy is relatively low. Jin et al. [20] established ELISA using cyst fluid as coated antigen to detect the Eg antibody in Uzbekistan sheep, confirming that the sensitivity and specificity of the method were relatively high, but it had cross-reaction with other cysticerci and Clonorshis sinensis infected serum.

Some researchers extracted natural antigen from Eg cyst fluid as the diagnostic antigen and established serological test to detect Eg antibodies [18,24]. Feng et al. [18] used dot-immunogold filtration assay (DIGFA) to evaluate purified Eg cyst fluid antigen $\mathrm{AgB}$ for its diagnostic effect for Eg and found the sensitivity and specificity of this method were $90.7 \%$ and $94.6 \%$, respectively. Furthermore, an $8 \mathrm{kDa}$ purified cyst fluid protein $\mathrm{AgB}$ for its diagnostic value for sheep $\mathrm{CE}$ was evaluated. The results showed that the diagnostic sensitivity and specificity of purified $8 \mathrm{kDa}$ protein was beyond 90\% [27]. However, so far, there is no standard purification procedure that can be used to effectively prepare large scale standard $8 \mathrm{kDa}$ $\mathrm{AgB}$ protein, which limits the application of $\mathrm{AgB}$ antigen in $\mathrm{CE}$ serological diagnosis [28].

Recently, serological tests based on recombinant protein such as $\mathrm{Eg} \mathrm{AgB}$ and $\mathrm{Eg} 95$ has been developed in CE serological diagnosis $[13,29]$. DIGFA based on recombinant protein AgB (reAgB) for the detection of Eg antibody confirmed that reAgB had higher specificity but lower sensitivity than the crude purified $\mathrm{AgB}$ [25]. To compensate for weak reactionogenicity of single antigen, multi-epitope antigens based on dominant epitopes were explored in recent years. As a new strategy, the dominant epitopes of different antigens can be combined to generate multi-epitope antigens, which has been utilized to improve the antigenicity of proteins. Jiang et al. [14] predicted the structures of $\mathrm{Eg} \mathrm{AgB1}, \mathrm{AgB} 2$, and $\mathrm{AgB} 4$ subunits using the online software and constructed recombinant multi-epitope antigens. The results showed that the reactionogenicity of recombinant multi-epitope proteins is stronger than that of single $\mathrm{AgB}$ subunit. In this study, the developed ELISA based on the Eg mefAg-1 displayed high agreement with the commercial anti-Echinococcus IgG ELISA Kit, which also confirmed that constructing multiple epitope antigens was useful strategy to improve reactionogenicity of diagnostic antigens.

With the accomplishment of complete genome sequence of some tapeworms [30,31], many important antigens of Eg were analyzed and predicted with serological diagnosis potential, such as antigen B (AgB, EGR_06805), tetraspanin (TSP1, EGR_10196; TSP6, EGR_00715), reticulon (RTN, EGR_04657), protease inhibitors (PI, EGR_03566) and Eg95 (EGR_05614) [32]. On the basis of these studies, we developed multi-epitope fusion CDNA Eg mefAg-1 and prepared Eg mefAg-1. The recombinant antigen Eg mefAg-1 had no cross-reaction with sera against other parasites in sheep in the same sites. The sensitivity of $94.38 \%$ and specificity of $98.63 \%$ were observed in the indirect ELISA based on Eg mefAg-1, which shared high agreement with necropsy, the gold standard recommended by OIE Manual. Our results demonstrated that Eg mefAg-1 had high serodiagnostic performance, which may be a potentially valuable candidate for CE serodiagnosis in sheep.

\section{ACKNOWLEDGMENTS}

We thank the field staff who provided the technical assistance for this study. This work was supported by Grant from National Key Research and Development Program (No.2017YFD0501200), International Science \& Technology Cooperation Program of XPCC (No. 2016AH006) and Xinjiang Autonomous Region graduate innovation project (No. XJGRI2014059). The experiments were carried out in accordance with the guidelines issued by the Ethical Committee of Shihezi University.

\section{CONFLICT OF INTEREST}

The authors declare that they have no conflict of interest.

\section{REFERENCES}

1. Siracusano A, Riganò R, Ortona E, Profumo E, Margutti P, Buttari B, Delunardo F, Teggi A. Immunomodulatory mechanisms during Echinococcus granulosus infection. Exp Parasitol 2008; 119: 483-489.

2. Craig P, Mastin A, van Kesteren F, Boufana B. Echinococcus granulosus: epidemiology and state-of-the-art of diagnostics in animals. Vet Parasitol 2015; 213: 132-148.

3. Hidalgo C, García MP, Stoore C, Ramírez JP, Monteiro KM, Hell- 
man U, Zaha A, Ferreira HB, Galanti N, Landerer E, Paredes R. Proteomics analysis of Echinococcus granulosus protoscolex stage. Vet Parasitol 2016; 218: 43-45.

4. Budke CM, White AC Jr, Garcia HH. Zoonotic larval cestode infections: neglected, neglected tropical diseases? PLoS Negl Trop Dis 2009; 3: e319.

5. Marcinkutė A, Šarkūnas M, Moks E, Saarma U, Jokelainen P, Bagrade G, Laivacuma S, Strupas K, Sokolovas V, Deplazes P. Echinococcus infections in the Baltic region. Vet Parasitol 2015; 213: 121-131.

6. Qingling M, Guanglei W, Jun Q, Xinquan Z, Tianli L, Xuemei S, Jinsheng Z, Huisheng W, Kuojun C, Chuangfu C. Prevalence of hydatid cysts in livestock animals in Xinjiang, China. Korean J Parasitol 2014; 52: 331-334.

7. Li T, Chen X, Zhen R, Qiu J, Qiu D, Xiao N, Ito A, Wang H, Giraudoux P, Sako Y, Nakao M, Craig PS. Widespread co-endemicity of human cystic and alveolar echinococcosis on the eastern Tibetan Plateau, northwest Sichuan/southeast Qinghai, China. Acta Trop 2010; 113: 248-256.

8. Ma J, Wang H, Lin G, Zhao F, Li C, Zhang T, Ma X, Zhang Y, Hou Z, Cai H, Liu P, Wang Y. Surveillance of echinococcus isolates from Qinghai, China. Vet Parasitol 2015; 207: 44-48.

9. Guo Z, Li W, Peng M, Duo H, Shen X, Fu Y, Irie T, Gan T, Kirino Y, Nasu T, Horii Y, Nonaka N. Epidemiological study and control trial of taeniid cestode infection in farm dogs in Qinghai Province, China. J Vet Med Sci 2014; 76: 395-400.

10. Zhang W, Zhang Z, Wu W, Shi B, Li J, Zhou X, Wen H, McManus DP. Epidemiology and control of echinococcosis in central Asia, with particular reference to the People's Republic of China. Acta Trop 2015; 141: 235-243.

11. Carmena D, Cardona GA. Echinococcosis in wild carnivorous species: epidemiology, genotypic diversity, and implications for veterinary public health. Vet Parasitol 2014; 202: 69-94.

12. Agudelo Higuita NI, Brunetti E, McCloskey. Cystic echinococcosis. J Clin Microbiol 2016; 54: 518-523.

13. Kalantari E, Bandehpour M, Pazoki R, Taghipoor-Lailabadi N, Khazan H, Mosaffa N, Nazaripouya MR, Kazemi B. Application of recombinant Echinococcus granulosus antigen B to ELISA kits for diagnosing hydatidosis. Parasitol Res 2010; 106: 847-851.

14. Jiang L, Zhang YG, Liu MX, Feng Z. Analysis on the reactivity of five subunits of antigen B family in serodiagnosis of echinococcosis. Exp Parasitol 2012; 131: 85-91.

15. Jiao W, Fu C, Liu WL, Chai JJ. Preliminary evaluation on the diagnostic performance of five native antigens from Echinococcus granulosus. Zhongguo Ji Sheng Chong Xue Yu Ji Sheng Chong Bing Za Zhi 2013; 31: 357-362 (In Chinese).

16. Brunetti E, Kern P, Vuitton DA. Expert consensus for the diagnosis and treatment of cystic and alveolar echinococcosis in humans. Acta Trop 2010; 114: 1-16.

17. Golassa L, Abebe T, Hailu A. Evaluation of crude hydatid cyst fluid antigens for the serological diagnosis of hydatidosis in cattle. J Helminthol 2011; 85: 100-108.

18. Feng X, Wen H, Zhang Z, Chen X, Ma X, Zhang J, Qi X, Brad- shaw H, Vuitton D, Craig PS. Dot immunogold filtration assay (DIGFA) with multiple native antigens for rapid serodiagnosis of human cystic and alveolar echinococcosis. Acta Trop 2010; 113: 114-120.

19. Zhang W, Wen H, Li J, Lin R, McManus DP. Immunology and immunodiagnosis of cystic echinococcosis: an update. Clin Dev Immunol 2012; 2012: 101895.

20. Jin Y, Anvarov K, Khajibaev A, Hong S, Hong ST. Serodiagnosis of echinococcosis by ELISA using cystic fluid from Uzbekistan sheep. Korean J Parasitol 2013; 51: 313-317.

21. Bauomi IR, El-Amir AM, Fahmy AM, Zalat RS, Diab TM. Evaluation of purified $27.5 \mathrm{kDa}$ protoscolex antigen-based ELISA for the detection of circulating antigens and antibodies in sheep and human hydatidosis. J Helminthol 2015; 89: 577-583.

22. Hassanain MA, Shaapan RM, Khalil FA. Sero-epidemiological value of some hydatid cyst antigen in diagnosis of human cystic echinococcosis. J Parasit Dis 2016; 40: 52-56.

23. Iraqi W. Diagnostic value of semi-purified antigens of hydatid cyst fluid in human cystic echinococcosis. Acta Parasitol 2016; 61: 144-150.

24. Reiterová K, Auer H, Altintaś N, Yolasigmaz A. Evaluation of purified antigen fraction in the immunodiagnosis of cystic echinococcosis. Parasitol Res 2014; 113: 2861-2867.

25. Chen X, Chen X, Lu X, Feng X, Wen H. The production and comparative evaluation of native and recombinant antigens for the fast serodiagnosis of cystic echinococcosis with dot immunogold filtration assay. Parasite Immunol 2015; 37: 10-15.

26. Ma X, Zhou X, Zhu Y, Li Y, Wang H, Mamuti W, Li Y, Wen H, Ding J. The prediction of T- and B-combined epitope and tertiary structure of the Eg95 antigen of Echinococcus granulosus. Exp Ther Med 2013; 6: 657-662.

27. Jeyathilakan N, Abdul Basith S, John L, Chandran ND, Raj GD. Development and evaluation of flow through technique for diagnosis of cystic echinococcosis in sheep. Vet Parasitol 2011; 180: 250-255.

28. Manzano-Román R, Sánchez-Ovejero C, Hernández-González A, Casulli A, Siles-Lucas M. Serological diagnosis and follow-up of human cystic echinococcosis: a new hope for the future? Biomed Res Int 2015; 2015: 428205.

29. Rouhani S, Parvizi P, Spotin A. Using specific synthetic peptide (p176) derived AgB 8/1-kDa accompanied by modified patient's sera: a novel hypothesis to follow-up of cystic echinococcosis after surgery. Med Hypotheses 2013; 81: 557-560.

30. Tsai IJ, Zarowiecki M, Holroyd N, Garciarrubio A, SánchezFlores A, Brooks KL, Tracey A, Bobes RJ, Fragoso G, Sciutto E, Aslett M, Beasley H, Bennett HM, Cai J, Camicia F, Clar R, Cucher M, De Silva N, Day TA, Deplazes P, Estrada K, Fernández C, Holland PW, Hou J, Hu S, Huckvale T, Hung SS, Kamenetzky L, Keane JA, Kiss F, Koziol U, Lambert O, Liu K, Luo X, Luo Y, Macchiaroli N, Nichol S, Paps J, Parkinson J, Pouchkina-Stantcheva N, Riddiford N, Rosenzvit M, Salinas G, Wasmuth JD, Zamanian M, Zheng Y, Cai J, Soberón X, Olson PD, Laclette JP, Brehm K, Berriman $\mathrm{M}$. The genomes of four tapeworm species reveal adapta- 
tions to parasitism. Nature 2013; 496: 57-63.

31. Zhang W, Wang S, McManus DP. Echinococcus granulosus genomics: a new dawn for improved diagnosis, treatment, and control of echinococcosis. Parasite 2014; 21: 66.

32. Zheng H, Zhang W, Zhang L, Zhang Z, Li J, Lu G, Zhu Y, Wang Y,
Huang Y, Liu J, Kang H, Chen J, Wang L, Chen A, Yu S, Gao Z, Jin L, Gu W, Wang Z, Zhao L, Shi B, Wen H, Lin R, Jones MK, Brejova B, Vinar T, Zhao G, McManus DP, Chen Z, Zhou Y, Wang $\mathrm{S}$. The genome of the hydatid tapeworm Echinococcus granulosus. Nat Genet 2013; 45: 1168-1175. 
